\title{
Oxidation of Tertiary Homoallylic Alcohols by Thallium Trinitrate: Fragmentation vs. Ring Contraction
}

\author{
Luiz F. Silva Jr., ${ }^{*, a}$ Samir A. P. Quintiliano, ${ }^{a}$ Helena M. C. Ferraz, ${ }^{a}$ Leonardo S. Santos $^{b}$ and \\ Marcos N. Eberlin \\ ${ }^{a}$ Instituto de Química, Universidade de São Paulo, CP 26077, 05513-970 São Paulo-SP, Brazil \\ ${ }^{b}$ Instituto de Química, Universidade Estadual de Campinas, 13083-970 Campinas-SP, Brazil
}

\begin{abstract}
A oxidação de álcoois homoalílicos terciários com trinitrato de tálio (TTN) foi investigada. Os álcoois que possuem uma metila na posição alílica perdem uma molécula de acetona via uma reação de fragmentação, levando a uma mistura de álcoois alílicos isoméricos como principais produtos, juntamente com os correspondentes derivados acetilados. Por outro lado, o tratamento com TTN de álcoois terciários análogos, sem a metila na posição alílica, fornece indanos, através de uma reação de contração de anel.
\end{abstract}

The oxidation of tertiary homoallylic alcohols with thallium trinitrate (TTN) was investigated. The alcohols bearing an allylic methyl group lose a molecule of acetone via a fragmentation reaction that leads to isomeric secondary allylic alcohols as major products, together with their corresponding acetylated derivatives. On the other hand, treating analogous tertiary alcohols without the allylic methyl group with TTN gives indans, through a ring contraction reaction.

Keywords: thallium trinitrate, ring contraction, homoallylic alcohols, fragmentation reaction, indan

\section{Introduction}

The reaction of primary homoallylic alcohols with thallium(III) salts has been carefully investigated by several groups, and these studies revealed four main different reaction pathways governed mainly by the structure of the substrate. ${ }^{1}$ The formation of cyclic ethers through an electrophilic cyclization has been the most explored reaction (Scheme 1, Reaction A). ${ }^{2-7}$ With homoallylic alcohols bearing an endocyclic double bond, oxidative rearrangement may lead to a ring contraction product (Scheme 1, Reaction B), ${ }^{6-9}$ as exemplified by the preparation of the indan $\mathbf{2}$ from the alkenol 1 using thallium trinitrate (TTN) (Scheme 2). ${ }^{9}$ We observed that in these rearrangements the hydroxyl group has an active role, facilitating the addition of the thallium(III) to the double bond. The third type of reaction reported between thallium(III) and 3-alkenols proceeds via fragmentation, in which a molecule of formaldehyde is lost (Scheme 1, Reaction C). ${ }^{10,11}$ This reaction was discovered by Kocovský and Baines in the early 90's, resulting in an efficient approach to obtain the hormones estrone and

*e-mail: luizfsjr@iq.usp.br estradiol. ${ }^{12,13}$ Finally, homoallylic alcohols can afford a diastereomeric mixture of products of addition of the solvent when treated with thallium(III) in the presence of methanol (Scheme 1, Reaction D).,

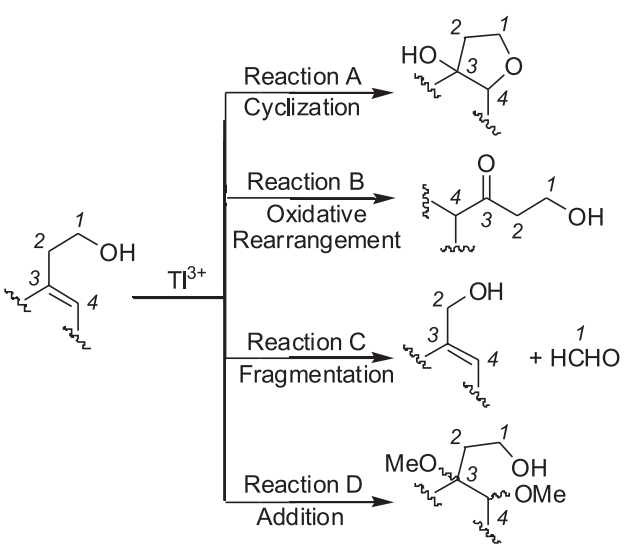

Scheme 1.

Although the reactivity of several primary homoallylic alcohols has been investigated, the behavior of the corresponding tertiary alcohols has not been studied. Thus, we decided to investigate the reactivity of a series of tertiary homoallylic alcohols analogous to $\mathbf{1}$, with 


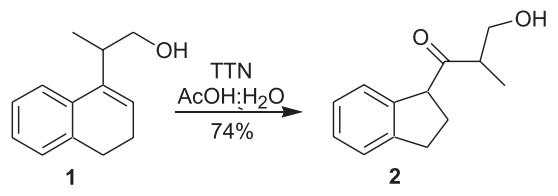

Scheme 2.

thallium(III). However, as described herein, the oxidation of these substrates with TTN can give either fragmentation or ring contraction products depending on the structure of the substrate.

\section{Results and Discussion}

The required tertiary homoallylic alcohols $\mathbf{4 a - g}$ were prepared in good yields from the reaction of the esters 3a-g ${ }^{9}$ with excess of methyllithium (Table 1). ${ }^{14}$

Treatment of the tertiary homoallylic alcohols $\mathbf{4 a - b}$ with TTN, under conditions similar to those used in the ring contraction of $\mathbf{1}$, failed to form the expected indans. Instead, the isomeric primary allylic alcohols $\mathbf{5 a - b}$ and $\mathbf{6} \mathbf{a}-\mathbf{b}$ were obtained, together with minor amounts of their acetylated derivatives 7a-b and 8a-b. Presumably, a molecule of acetone is lost in these fragmentations (Table 2, entries 1 and 2).

The different reactivity of $\mathbf{4 a - b}$ when compared to $\mathbf{1}$ can be explained considering the mechanism for the thallium(III) mediated ring contraction of homoallylic alcohols. In the rearrangement of $\mathbf{1}$, the coordination of the thallium(III) with the hydroxyl group leads to the formation of a heterocyclic six-membered ring intermediate. However, we believe that the formation of an analogous intermediate (10) from the substrates $\mathbf{4 a - b}$ would be sterically hindered, due to the presence of three methyl groups too close to each other, on which two of them would be in an axial position, as exemplified for $\mathbf{4 a}$ in Scheme 3.

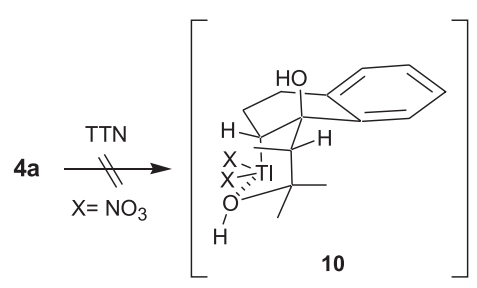

Scheme 3.

The behavior of $\mathbf{4} \mathbf{c}$ in the oxidation with TTN is slightly different from $\mathbf{4 a - b}$, because the indan $9 \mathbf{c}$ was isolated in addition to the fragmentation products (Table 2, entry 3 ). The formation of $\mathbf{9 c}$ can be rationalized as a result of the thallium(III) mediated oxidative rearrangement of the fragmentation product $\mathbf{5 c}$, which bears a methoxyl group at the para position of the migrating carbon $8 \mathrm{a}$, increasing its migratory aptitude and, thus, facilitating the ring contraction (Scheme 4). ${ }^{17,18}$ In the analogous allylic alcohols 5a-b this
Table 1. Preparation of the tertiary alcohols $\mathbf{4 a - \mathbf { g } ^ { \mathrm { a } }}$<smiles>CCOC(=O)C(C)C1=CCCc2ccccc2C1=CCC(C)C(C)C(=O)OCC</smiles>

3<smiles>CCOC(=O)C(C)C1=CCCc2cc(OC)ccc21</smiles><smiles>COc1ccc2c(c1)CCC=C2C(C)C(C)(C)O</smiles>

4<smiles>CCOC(=O)CC1=CCCc2ccccc21</smiles><smiles>CC(C)(O)CC1=CCCc2c1cccc2C(C)(C)C</smiles>

5<smiles>CCOC(=O)CC1=CCCc2ccc(OC)cc21</smiles><smiles>COc1cc2c(cc1[14CH3])C(CC(C)(C)O)=CCC2</smiles>

6<smiles>CCOC(=O)CC1=CCCc2cc(OC)ccc21</smiles><smiles>COc1ccc2c(c1)CCC=C2CC(C)(C)O</smiles>

7<smiles>CCOC(=O)CC1=CCC(C)c2ccccc21</smiles><smiles>CC(C)(O)CC1=CCC([Ge])c2ccccc21</smiles>

${ }^{a}$ Reaction conditions: 6 equiv. of MeLi, THF, $-78^{\circ} \mathrm{C}, 2 \mathrm{~h}$.

mesomeric effect can not operate. To corroborate this proposition, the reaction of $\mathbf{4} \mathbf{c}$ was performed using enough TTN to consume all 5c formed. Indeed, under such a condition, the indan 9c was obtained in $42 \%$ yield (Table 2, entry 4).

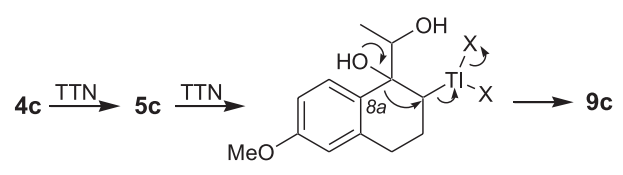

Scheme 4. 


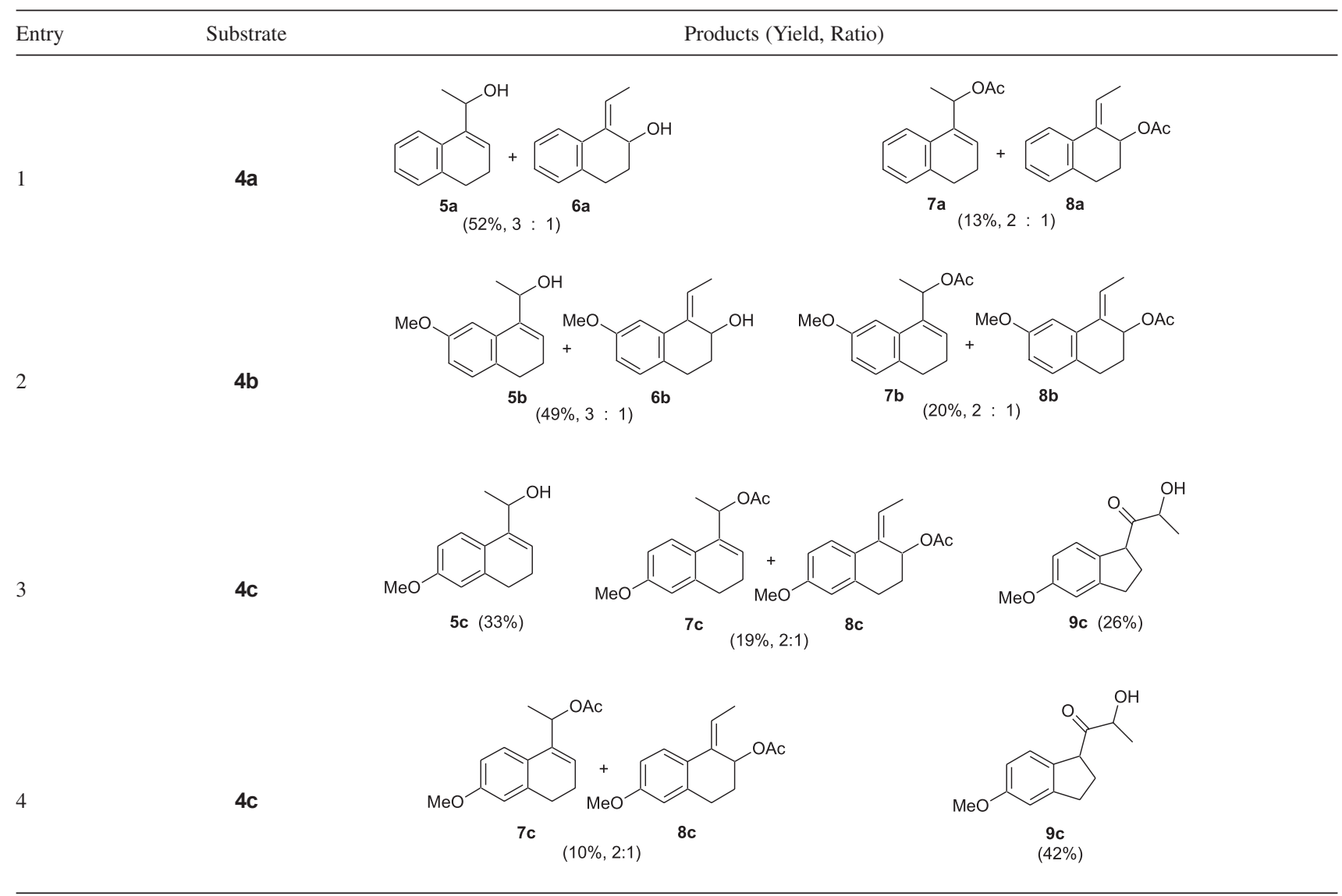

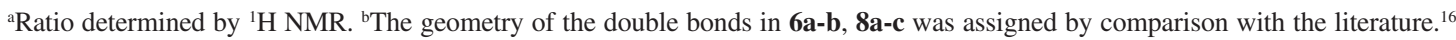

Three possible mechanisms can be invoked for explaining the fragmentation reaction of the alcohols $\mathbf{4 a}$ c. First, the mechanism previously proposed by Kocovský and Baines ${ }^{13}$ for the thallium(III) promoted fragmentation reactions was applied for the homoallylic alcohol $\mathbf{4 a}$ (Scheme 5, path A). The first step is an anti-Markovnikov electrophilic addition of the thallium(III), forming the cyclic ether 11. This step also represents a 5-endo-trig ring closure, which is usually not favored. Reorganization of the bonds would lead to the loss of acetone, affording the allylic carbocation 12, which after solvolysis gives the final products $\mathbf{5 a - 8}$. Considering that the course of several thallium(III) mediated cyclization reactions had been well explained by an initial 4-exo-trig cyclization, which proceeded by a Markovnikov addition of thallium(III), ${ }^{4}$ we set out to expand this feature in the Kocovský mechanism through a new sequence of events (Scheme 5, path B). As the thallium(III) is isoeletronic to $\mathrm{Pb}(\mathrm{IV})$ and based on the recent work of Preite and Cuellar, ${ }^{19}$ the thallium(III) $\pi$-allylic complex $\mathbf{1 6}$ was postulated as the key intermediate in the third hypothesis (Scheme 5, path C).
Instead of the fragmentation products observed in the oxidation of the alcohols $\mathbf{4 a - c}$, the substrates $\mathbf{4 d - g}$ gave the ring contraction products $\mathbf{1 7} \mathbf{d}-\mathbf{g}$ when treated with TTN (Table 3). Furthermore, these ring contraction products 17d-g were obtained in higher yields using 1.5 equiv. (59$65 \%$ ) than using 1.1 equiv. of TTN (37-60\%). This can be explained by the fact that the ring contraction is faster when the amount of the oxidizer is increased. Thus, the formation of byproducts is reduced, giving higher isolated yields for 17d-g. In entries 2 and 4, the starting material was not totally consumed, even with 1.5 equiv. of TTN. Thus, the alcohol $\mathbf{4 g}$ was treated with 1.7 equivalents of TTN expecting that the yield could be further increased. However, albeit no starting material was recovered, the yield was lower (59\%) than that with 1.5 equivalents $(65 \%)$. We consider that the additional excess of TTN oxidizes the ketone moiety of the indan $\mathbf{1 7 g}$. The indan $\mathbf{1 7} \mathrm{g}$ was obtained exclusively in the trans configuration, which agrees with a similar result previously described. ${ }^{9}$

In summary, the reaction with TTN of the tertiary homoallylic alcohols 4a-c, which bear an allylic methyl group, fails to form the expected ring contraction products. 
Table 3. Reaction of $\mathbf{4 d - g}$ with 1.5 equiv. of TTN in AcOH: $\mathrm{H}_{2} \mathrm{O}(2: 1)$, rt Entry Substrate Product (Yield) $)^{15}$

1 $4 d$<smiles>CC(C)(O)CC(=O)C1CCc2ccccc21</smiles>

2 4 e<smiles>COc1ccc2c(c1)CCC2C(=O)CC(C)(C)O</smiles>

$4 f$<smiles>COc1ccc2c(c1)CCC2C(=O)CC(C)(C)O</smiles>

4

$4 \mathrm{~g}$

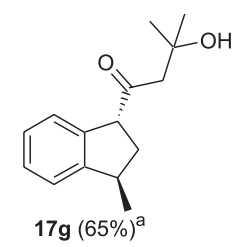

${ }^{a} 4 \%$ of starting material were recovered on each case.

Instead, the observed products are those originated from a fragmentation reaction. On the other hand, treating analogous tertiary alcohols without the allylic methyl group (4d-g) with TTN gives the corresponding indans (17d-g), in good yield, through a ring contraction reaction.

\section{Experimental}

\section{General}

THF was freshly distilled from sodium/benzophenone. Other reagents were used as received. Column chromatography was performed using silica gel Acros 200-400 Mesh. TLC analyses were performed with silica gel plates Merck, using $p$-anisaldehyde solution for visualization. ${ }^{1} \mathrm{H}$ and ${ }^{13} \mathrm{C}$ NMR spectra were recorded on Bruker spectrometers. IR spectra were measured on a PerkinElmer 1750-FT. Gas chromatography analyses were performed in a HP-6890 series II. High resolution mass spectra were performed on a VG Autospec/Fission Instrument and MicroTOF LC from Bruker Daltonics.

3-(1,2-Dihydronaphthalen-4-yl)-2-methylbutan-2-ol (4a). General procedure for the preparation of the 3-alkenols $4 a-g$

To a stirred solution of $3 \mathbf{a}^{9}(0.975 \mathrm{~g}, 4.24 \mathrm{mmol})$ in anhydrous THF (10 mL), was added MeLi (17.0 mL of $1.6 \mathrm{~mol} \mathrm{~L}^{-1}$ solution in $\mathrm{Et}_{2} \mathrm{O}, 25.4 \mathrm{mmol}$ ) at $-78{ }^{\circ} \mathrm{C}$. The mixture was stirred at $-78{ }^{\circ} \mathrm{C}$ for $2 \mathrm{~h}$, when water was carefully added dropwise at rt. The aqueous phase was extracted twice with AcOEt. The combined organic phase was washed with brine and dried over anhydrous $\mathrm{MgSO}_{4}$. The solvent was removed under reduced pressure. The residue was purified by flash chromatography (gradient elution, 10-20\% AcOEt in hexanes) giving impure 3-(1,2-dihydronaphthalen-4-yl)butan-2one as byproduct $(0.073 \mathrm{~g})$ and 3-(1,2-dihydronaphthalen-4-yl)-2-methylbutan-2-ol (4a) (0.436 g, $2.02 \mathrm{mmol}, 48 \%$ ); colorless oil; IR (film) $\mathrm{v}_{\max } / \mathrm{cm}^{-1}: 712$, 942, 1453, 3444; ${ }^{1} \mathrm{H}$ NMR (300 MHz, $\left.\mathrm{CDCl}_{3}\right) \delta 1.02$ (s, 3H), 1.12 (s, 3H), 1.13 (d, J 7.2 Hz, 3H), 1.63 (br s, $1 \mathrm{H}), 2.16-2.37(\mathrm{~m}, 2 \mathrm{H}), 2.70(\mathrm{dd}, J 6.3$ and $9.3 \mathrm{~Hz}$,

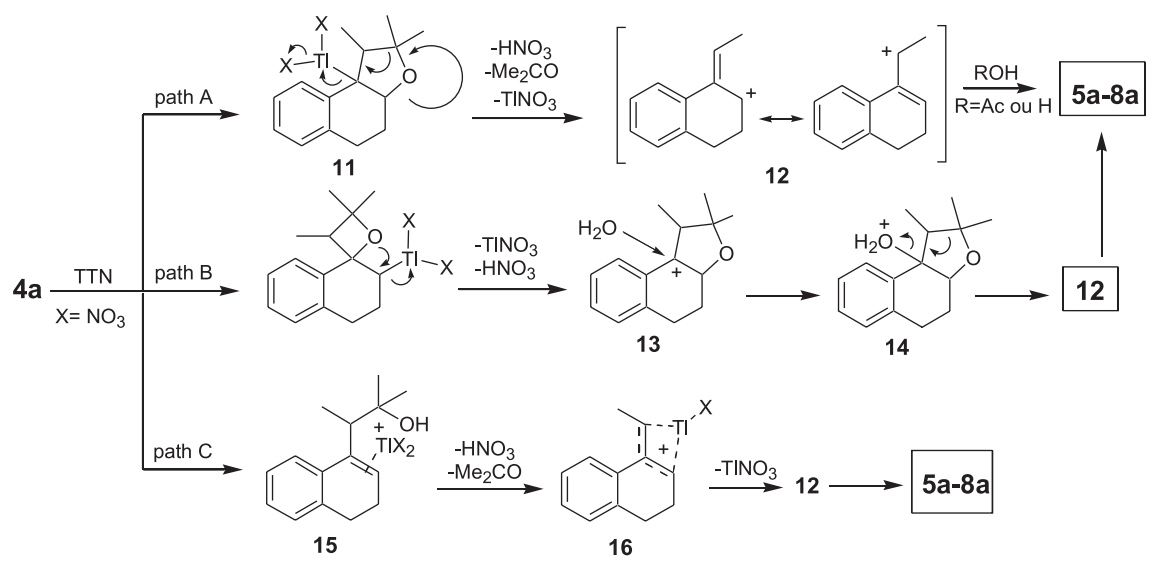

Scheme 5. 
2H), $3.10(\mathrm{q}, J 7.5 \mathrm{~Hz}, 1 \mathrm{H}), 6.03(\mathrm{dd}, J 3.6$ and $5.6 \mathrm{~Hz}$, $1 \mathrm{H}), 7.09-7.40(\mathrm{~m}, 3 \mathrm{H}), 7.42(\mathrm{~d}, J 7.5 \mathrm{~Hz}, 1 \mathrm{H}) ;{ }^{13} \mathrm{C}$ NMR $\left(75 \mathrm{MHz}, \mathrm{CDCl}_{3}\right) \delta 15.7,23.0,27.4,28.7,28.8$, 42.3, 72.7, 122.8, 125.6, 126.2, 126.5, 127.6, 136.2, 136.6, 139.8; LRMS (EI) $\mathrm{m} / z$ (rel. int.) $216\left(\mathrm{M}^{+}, 2 \%\right)$, 158 (70), 129 (100). Anal. calc. for $\mathrm{C}_{15} \mathrm{H}_{20} \mathrm{O}$ : C, 83.28; H, 9.32. Found: C, 83.49; H, 9.24.

3-(1,2-Dihydro-6-methoxynaphthalen-4-yl)-2-methylbutan$2-o l(4 b)$. The reaction was performed as described above, using $3 \mathbf{b}^{9}$ (1.03 g, $\left.3.97 \mathrm{mmol}\right)$, THF (10 mL) and MeLi $(18.0 \mathrm{~mL}, 28.8 \mathrm{mmol})$. The residue was purified by flash chromatography (gradient elution, 15-30\% AcOEt in hexanes) giving impure 3-(1,2-dihydro-6-methoxynaphthalen-4-yl)butan-2-one as byproduct $(0.091 \mathrm{~g})$ and 3-(1,2-dihydro-6-methoxynaphthalen-4-yl)-2-methylbutan2-ol (4b) (0.745 g, $3.03 \mathrm{mmol}, 76 \%)$; pale yellow oil; IR (film) $v_{\max } / \mathrm{cm}^{-1}: 944,1163,1246,3457 ;{ }^{1} \mathrm{H} N M R(300 \mathrm{MHz}$, $\left.\mathrm{CDCl}_{3}\right) \delta 1.11(\mathrm{~s}, 3 \mathrm{H}), 1.21(\mathrm{~s}, 3 \mathrm{H}), 1.23(\mathrm{~d}, J 6.6 \mathrm{~Hz}, 3 \mathrm{H})$, 2.13-2.32 (m, 3H), 2.59-2.65 (m, 2H), $3.02(\mathrm{q}, J 7.2 \mathrm{~Hz}$, $1 \mathrm{H}), 3.78(\mathrm{~s}, 3 \mathrm{H}), 6.05(\mathrm{dd}, J 3.9$ and $5.4 \mathrm{~Hz}, 1 \mathrm{H}), 6.67(\mathrm{dd}$, $J 2.4$ and $8.1 \mathrm{~Hz}, 1 \mathrm{H}), 7.00(\mathrm{~d}, J 2.1 \mathrm{~Hz}, 1 \mathrm{H}), 7.05(\mathrm{~d}, J 8.4$ $\mathrm{Hz}, 1 \mathrm{H}) ;{ }^{13} \mathrm{C} \mathrm{NMR}\left(75 \mathrm{MHz}, \mathrm{CDCl}_{3}\right) \delta 15.7,23.4,27.5$, 27.7, 28.8, 42.4, 55.2, 72.6, 110.0, 110.6, 126.2, 128.1, 128.7, 137.3, 139.6, 158.2; LRMS (EI) $\mathrm{m} / z$ (rel. int.) 246 $\left(\mathrm{M}^{+}, 2 \%\right), 188$ (91), 159 (100). Anal. calc. for $\mathrm{C}_{16} \mathrm{H}_{22} \mathrm{O}_{2}: \mathrm{C}$, 78.01; H, 9.00. Found: C, 78.00; H, 8.64.

3-(1,2-Dihydro-7-methoxynaphthalen-4-yl)-2-methylbutan$2-o l(4 c)$. The reaction was performed as described above, using $3 \mathbf{c}^{9}(1.03 \mathrm{~g}, 3.97 \mathrm{mmol})$, THF (10 mL) and MeLi (15.0 $\mathrm{mL}, 24.0 \mathrm{mmol})$. The residue was purified by flash chromatography (gradient elution, 15-30\% AcOEt in hexanes) giving impure 3-(1,2-dihydro-7-methoxynaphthalen-4-yl)butan-2-one (0.060 g) and 3-(1,2-dihydro7-methoxynaphthalen-4-yl)-2-methylbutan-2-ol (4c) $(0.766$ g, $3.11 \mathrm{mmol}, 78 \%$ ); pale yellow oil; IR (film) $v_{\max } / \mathrm{cm}^{-1}$ : 943, 1201, 1250, 3455; ${ }^{1} \mathrm{H}$ NMR (300 MHz, $\left.\mathrm{CDCl}_{3}\right) \delta 1.08$ (s, 3H), 1.18 (s, 3H), 1.19 (d, J 6.2 Hz, 3H), 1.84 (br s, 1H), 2.14-2.30 (m, 2H), $2.64(\mathrm{dd}, J 6.3$ and $9.3 \mathrm{~Hz}, 2 \mathrm{H}), 3.01(\mathrm{q}$, $J 7.2 \mathrm{~Hz}, 1 \mathrm{H}), 3.76(\mathrm{~s}, 3 \mathrm{H}), 5.85$ (t, $J 6.0 \mathrm{~Hz}, 1 \mathrm{H}), 6.66-6.71$ (m, 2H), $7.31(\mathrm{~d}, J 9.3 \mathrm{~Hz}, 1 \mathrm{H}) ;{ }^{13} \mathrm{C} \mathrm{NMR}\left(75 \mathrm{MHz}, \mathrm{CDCl}_{3}\right)$ $\delta$ 15.9, 23.2, 27.5, 29.1, 29.4, 42.6, 55.2, 72.8, 110.9, 113.9, 123.3, 124.3, 129.5, 138.5, 139.5, 158.3; LRMS (EI) $\mathrm{m} / \mathrm{z}$ (rel. int.) $246\left(\mathrm{M}^{+}, 19 \%\right), 188$ (90), 59 (100); HRMS [ESI $(-)]$ Calc. for $\left[\mathrm{C}_{16} \mathrm{H}_{22} \mathrm{O}_{2}-\mathrm{H}\right]^{-}$245.1542. Found: 245.1542.

\section{1-(1,2-Dihydronaphthalen-4-yl)-2-methylpropan-2-ol} $(4 d)$. The reaction was performed as described above, using $3 \mathbf{d}^{9}$ (2.52 g, $\left.11.7 \mathrm{mmol}\right)$, THF (25.0 $\mathrm{mL}$ ) and MeLi $(43.8 \mathrm{~mL}, 70.0 \mathrm{mmol})$. The residue was purified by flash chromatography (gradient elution, 15-30\%, AcOEt in hexanes) giving impure 1-(1,2-dihydronaphthalen-4yl)propan-2-one $(0.0730 \mathrm{~g})$ and the alcohol $4 \mathbf{d}(1.29 \mathrm{~g}$, $8.17 \mathrm{mmol}, 55 \%)$; white solid; mp 72.1-73.2 ${ }^{\circ} \mathrm{C}$; IR ( $\left.\mathrm{KBr}\right)$ $v_{\max } / \mathrm{cm}^{-1}: 903,1129,1361,3331 ;{ }^{1} \mathrm{H}$ NMR (300 MHz, $\left.\mathrm{CDCl}_{3}\right) \delta 1.20$ (s, 6H), 1.61 (br s, $1 \mathrm{H}$ ), 2.27 (ddd, $J$ 4.6, 7.7 and $8.2 \mathrm{~Hz} 2 \mathrm{H}$ ), 2.70 (s, 2H), 2.75 (dd, $J 7.7$ and 8.2 $\mathrm{Hz}, 2 \mathrm{H}), 5.95$ (t, J 4.6 Hz, 1H), 7.12-7.21 (m, 3H), 7.36 $(\mathrm{d}, J 7.3 \mathrm{~Hz}, 1 \mathrm{H}) ;{ }^{13} \mathrm{C}$ NMR $\left(75 \mathrm{MHz}, \mathrm{CDCl}_{3}\right) \delta 23.3$, 28.8, 29.9, 45.1, 70.7, 123.7, 126.3, 126.8, 127.7, 129.8, 133.6, 135.3, 136.6; LRMS (EI) $\mathrm{m} / z$ (rel. int.) $202\left(\mathrm{M}^{+}\right.$, 10\%), 144 (80), 129 (100); Anal. calc. for $\mathrm{C}_{14} \mathrm{H}_{18} \mathrm{O}: \mathrm{C}$, 83.12; H, 8.97. Found: C, 83.15; H, 8.57.

1-(1,2-dihydro-6-methoxynaphthalen-4-yl)-2-methylpropan-2-ol $(4 \boldsymbol{e})$. The preparation was performed as described above, using $3 \mathrm{e}^{9}(0.832 \mathrm{~g}, 3.30 \mathrm{mmol})$, THF $(20 \mathrm{~mL})$ and $\mathrm{MeLi}(12.4 \mathrm{~mL}, 20.0 \mathrm{mmol})$. The residue was purified by flash chromatography (gradient elution, 15-30\% AcOEt in hexanes) giving impure 1-(1,2-dihydro6-methoxynaphthalen-4-yl)propan-2-one ( $0.101 \mathrm{~g})$ and the alcohol 4e (0.562 g, $2.42 \mathrm{mmol}, 73 \%)$; pale yellow oil; IR (film) $v_{\max } / \mathrm{cm}^{-1}: 976,1044,1607,3420 ;{ }^{1} \mathrm{H}$ NMR (300 $\mathrm{MHz}, \mathrm{CDCl}_{3}$ ) $\delta 1.17$ (s, 6H), 2.20 (ddd, J 4.5, 7.5 and 8.1 $\mathrm{Hz}, 2 \mathrm{H}$ ), 2.20 (br s, 1H), 2.63 (s, 2H), 2.63 (dd, J 6.3 and $9.6 \mathrm{~Hz}, 2 \mathrm{H}), 3.79$ (s, 3H), $5.97(\mathrm{t}, J 4.8 \mathrm{~Hz}, 1 \mathrm{H}), 6.68(\mathrm{dd}$, $J 2.7$ and $8.1 \mathrm{~Hz}, 1 \mathrm{H}), 6.94(\mathrm{~d}, J 2.7 \mathrm{~Hz}, 1 \mathrm{H}), 7.06(\mathrm{~d}, J$ $7.8 \mathrm{~Hz}, 1 \mathrm{H}) ;{ }^{13} \mathrm{C}$ NMR $\left(75 \mathrm{MHz}, \mathrm{CDCl}_{3}\right) \delta 23.6,27.7$, 29.7, 45.2, 55.2, 70.6, 110.6, 111.0, 128.1, 128.7, 130.3, 133.5, 136.4, 158.1; LRMS (EI) $\mathrm{m} / z$ (rel. int.) $232\left(\mathrm{M}^{+}\right.$, 14\%), 159 (81), 59 (100); Anal. calc. for $\mathrm{C}_{15} \mathrm{H}_{20} \mathrm{O}_{2}: \mathrm{C}$, 77.55; H, 8.68. Found: C, 77.37; H, 8.61.

1-(1,2-dihydro-7-methoxynaphthalen-4-yl)-2-methylpropan-2-ol $(4 f)$. The preparation was performed as described above, using $\mathbf{3 f}^{9}(0.786 \mathrm{~g}, 3.19 \mathrm{mmol})$, anhydrous THF (6.0 mL) and MeLi (12.0 mL, $19.2 \mathrm{mmol})$. The residue was purified by flash chromatography (gradient elution, 10-30\% AcOEt in hexanes) giving impure 1-(1,2-dihydro-7-methoxynaphthalen-4-yl)propan2-one $(0.097 \mathrm{~g})$ and the alcohol $4 \mathrm{f}(0.485 \mathrm{~g}, 2.09 \mathrm{mmol}$, 65\%); white solid; $\mathrm{mp} 84.6-86.0{ }^{\circ} \mathrm{C}$; IR (KBr) $v_{\max } / \mathrm{cm}^{-1}$ : 948, 1170, 1251, 3342; ${ }^{1} \mathrm{H}$ NMR (300 MHz, $\left.\mathrm{CDCl}_{3}\right)$ $\delta 1.19$ (s, 6H), 1.64 (br s, 1H), 2.26 (ddd, $J 4.8,7.5$ and $8.1 \mathrm{~Hz}, 2 \mathrm{H}), 2.66(\mathrm{~s}, 2 \mathrm{H}), 2.732(\mathrm{dd}, J 7.5$ and $8.1 \mathrm{~Hz}$, $2 \mathrm{H}), 3.78$ (s, 3H), 5.80 (t, J 4.2 Hz, 1H), 6.69-6.71 (m, $2 \mathrm{H}), 7.25-7.29(\mathrm{~m}, 1 \mathrm{H}) ;{ }^{13} \mathrm{C} \mathrm{NMR}\left(75 \mathrm{MHz}, \mathrm{CDCl}_{3}\right)$ $\delta$ 23.4, 29.4, 30.0, 45.4, 55.3, 70.8, 110.9, 114.0, 125.1, 127.3, 128.6, 133.4, 138.6, 158.5; LRMS (EI) $\mathrm{m} / \mathrm{z}$ (rel. int.) $232\left(\mathrm{M}^{+}, 15 \%\right), 174$ (100), 159 (85); Anal. calc. for $\mathrm{C}_{15} \mathrm{H}_{20} \mathrm{O}_{2}: \mathrm{C}, 77.55 ; \mathrm{H}, 8.68$. Found: C, 77.62; H, 8.68. 
1-(1,2-Dihydro-1-methylnaphthalen-4-yl)-2-methylpropan-2-ol $\mathbf{( 4 g )}$. The preparation was performed as described above, using $\mathbf{3 g}^{9}$ (1.003 g, $\left.4.36 \mathrm{mmol}\right)$, anhydrous THF (15 mL) and MeLi (16.3 mL, $26.2 \mathrm{mmol})$. The residue was purified by flash chromatography (gradient elution, 15-25\% AcOEt in hexanes) giving impure 1-(1,2-dihydro-1-methylnaphthalen-4-yl)propan2-one $(0.055 \mathrm{~g})$ and the alcohol 4g (0.602 g, $2.79 \mathrm{mmol}$, $64 \%$ ); colorless oil; IR (film) $v_{\max } / \mathrm{cm}^{-1}: 753,1140,3410$; ${ }^{1} \mathrm{H}$ NMR (300 MHz, $\left.\mathrm{CDCl}_{3}\right) \delta 1.20(\mathrm{~s}, 6 \mathrm{H}), 1.25$ (d, J 6.9 $\mathrm{Hz}, 3 \mathrm{H}$ ), 1.72 (br s, 1H), 2.10 (dddd, $J$ 0.8, 5.0, 7.2 and $16.9 \mathrm{~Hz}, 1 \mathrm{H}$ ), 2.46 (dddd, $J$ 0.9, $4.2,5.9$ and $17.0 \mathrm{~Hz}$, 1H), 2.64 (d, J $13.8 \mathrm{~Hz}, 1 \mathrm{H}), 2.76(\mathrm{~d}, J 13.9 \mathrm{~Hz}, 1 \mathrm{H})$, 2.88 (sx, J $6.9 \mathrm{~Hz}, 1 \mathrm{H}), 5.86$ (t, J 4.5 Hz, 1H), 7.16-7.19 $(\mathrm{m}, 3 \mathrm{H}), 7.36-7.39(\mathrm{~m}, 1 \mathrm{H}) ;{ }^{13} \mathrm{C} \mathrm{NMR}\left(75 \mathrm{MHz}, \mathrm{CDCl}_{3}\right)$ $\delta$ 19.9, 29.8, 31.2, 32.4, 45.1, 70.8, 123.8, 126.0, 126.3, 127.2, 128.1, 133.0, 134.4, 141.3; LRMS (EI) $\mathrm{m} / \mathrm{z}$ (rel. int.) $216\left(\mathrm{M}^{+}, 7 \%\right), 158(50), 143$ (100); HRMS [ESI(+)] Calc. for $\left[\mathrm{C}_{15} \mathrm{H}_{20} \mathrm{O}+\mathrm{Na}\right]^{+}:$255.1361. Found 255.1350.

\section{Oxidation of 4 a by 1.1 equiv. TTN. General Procedure for the Oxidation of $\mathbf{4 a}-\mathbf{g}$ with Thallium(III)}

To a stirred solution of $\mathbf{4 a}(0.323 \mathrm{~g}, 1.50 \mathrm{mmol})$ in $\mathrm{AcOH} / \mathrm{H}_{2} \mathrm{O}(2: 1,7.5 \mathrm{~mL})$ at $\mathrm{rt}$, was added TTN. $3 \mathrm{H}_{2} \mathrm{O}$ (0.731 g, $1.64 \mathrm{mmol})$, which promptly dissolved. The mixture was stirred for $1 \mathrm{~h}$ and an abundant precipitation was observed. The mixture was then filtrated through a silica gel pad $(10 \mathrm{~cm}, 200 \mathrm{~mL}$ of AcOEt as eluent) washed with the same volume of a saturated solution of $\mathrm{NaHCO}_{3}$. The aqueous phase was then extracted twice with AcOEt. The combined organic phase was washed with brine and dried over anhydrous $\mathrm{MgSO}_{4}$. The solvent was removed under reduced pressure and the residue was purified by flash chromatography (gradient elution, 15-40\% ethyl acetate in hexanes) giving a mixture of the alcohols $\mathbf{5 a}$ and $\mathbf{6 a}(3: 1,0.135 \mathrm{~g}, 0.776 \mathrm{mmol}, 52 \%)$ and of the esters 7a and 8a $(2: 1,0.041 \mathrm{~g}, 0.19 \mathrm{mmol}, 13 \%)$. Preparative TLC (1:1 AcOEt in hexanes) gave a 12:1 mixture of the alcohols $5 \mathbf{a}$ and $6 \mathbf{a}$. The synthesis of the compounds 7a and $\mathbf{8 a}$ has already been reported. ${ }^{16}$ The full characterization of these compounds is given below. Mixture of the isomeric alcohols 1-(1,2-dihydronaphthalen-4yl)ethanol (5a) and (Z)-1-ethylidene-1,2,3,4-tetrahydronaphthalen-2-ol (6a): Colorless oil; IR (film) $v_{\max } / \mathrm{cm}^{-1}$ : 739, 768, 1062, 1369, 2932, 3378; ${ }^{1} \mathrm{H}$ NMR (300 MHz, $\left.\mathrm{CDCl}_{3}\right) \delta$ (5a) 1.45 (d, J $\left.6.3 \mathrm{~Hz}, 3 \mathrm{H}\right), 1.7$ (br s, $\left.1 \mathrm{H}\right)$, 2.23-2.30 (m, 2H), 2.73 (t, J 8.1 Hz, 2H), 4.88 (dq, $J 1.2$ and $6.6 \mathrm{~Hz}, 1 \mathrm{H}), 6.17(\mathrm{dt}, J 0.9$ and $4.8 \mathrm{~Hz}, 1 \mathrm{H}), 7.13-$ 7.17 (m, 3H), 7.37 (d, J 7.5 Hz, 1H); (6a) 1.8 (br s, 1H), 1.97 (d, J 7.5 Hz, 3H), 2.17-2.24 (m, 2H), 2.92-3.06 (m,
2H), 5.00 (t, J 3.0 Hz, 1H), 6.27 (q, J 7.2 Hz, 1H), 7.11$7.23(\mathrm{~m}, 3 \mathrm{H}), 7.49-7.56(\mathrm{~m}, 1 \mathrm{H}) ;{ }^{13} \mathrm{C} \mathrm{NMR}(75 \mathrm{MHz}$, $\left.\mathrm{CDCl}_{3}\right) \delta$ (5a) 22.6, 22.7, 28.2, 67.2, 122.8, 123.7, 126.3, 126.7, 127.7, 133.2, 136.8, 140.4; (6a) 13.7, 24.3, 30.0, 63.9, 123.0, 123.8, 125.8, 126.2, 126.7, 128.7, 133.2, 136.2; LRMS (EI) m/z (rel. int.) $174\left(\mathrm{M}^{+}, 4 \%\right), 157$ (30), 129 (100); HRMS [ESI(-)] Calc. for $\left[\mathrm{C}_{12} \mathrm{H}_{14} \mathrm{O}-\mathrm{H}\right]^{-}$ 173.0966. Found: 173.0946. Mixture of the isomeric esters 1-(1,2-dihydronaphthalen-4-yl)ethyl acetate (7a) and (Z)-1-ethylidene-1,2,3,4-tetrahydronaphthalen-2-yl acetate (8a): Pale yellow oil; IR (film) $v_{\max } / \mathrm{cm}^{-1}: 1041$, 1241, 1449, 1735; ${ }^{1} \mathrm{H}$ NMR (300 $\left.\mathrm{MHz}, \mathrm{CDCl}_{3}\right) \delta(\mathbf{7 a})$ 1.38 (d, J 6.6 Hz, 3H), 1.97 (s, 3H), 2.15-2.22 (m, 2H), 2.60-2.66 (m, 2H), $5.83(\mathrm{dq}, J 0.9$ and $6.6 \mathrm{~Hz}, 1 \mathrm{H}), 6.07$ (dt, $J 0.6$ and $4.5 \mathrm{~Hz}, 1 \mathrm{H}), 7.02-7.06(\mathrm{~m}, 3 \mathrm{H}), 7.19(\mathrm{~d}, J$ $6.9 \mathrm{~Hz}, 1 \mathrm{H})$; (8a) 1.80 (d, J $7.5 \mathrm{~Hz}, 3 \mathrm{H}), 1.91$ (s, 3H), 2.56-2.58 (m, 2H), 2.95-2.83 (m, 2H), $6.00(\mathrm{t}, J 3.6 \mathrm{~Hz}$, $1 \mathrm{H}), 6.20$ (q, J $6.9 \mathrm{~Hz}, 1 \mathrm{H}), 6.98-7.10(\mathrm{~m}, 3 \mathrm{H}), 7.42-$ $7.45(\mathrm{~m}, 1 \mathrm{H}) ;{ }^{13} \mathrm{C} \mathrm{NMR}\left(75 \mathrm{MHz}, \mathrm{CDCl}_{3}\right) \quad \delta(\mathbf{7 a}) 19.8$, 21.4, 22.8, 28.0, 69.8, 122.8, 125.2, 126.1, 126.4, 126.9, 127.8, 132.8, 136.7, 170.4; (8a) 13.9, 21.2, 24.8, 27.8, $67.0,123.8,125.0,126.3,126.8,128.6,133.1,133.9$, 135.7, 170.6; LRMS (EI) $\mathrm{m} / z$ (rel. int.) $216\left(\mathrm{M}^{+}, 3 \%\right)$, 156 (59), 141 (100); HRMS [ESI(+)] Calc. for $\left[\mathrm{C}_{14} \mathrm{H}_{16} \mathrm{O}_{2}\right.$ $+\mathrm{H}]^{+}$217.1228. Found: 217.1231.

Oxidation of $\mathbf{4 b}$ by 1.1 equiv. TTN. The oxidation was performed following the general procedure, but using 4b (0.105 g, $0.427 \mathrm{mmol}), \mathrm{AcOH} / \mathrm{H}_{2} \mathrm{O}(2: 1,2.1 \mathrm{~mL})$ and TTN. $3 \mathrm{H}_{2} \mathrm{O}(0.209 \mathrm{~g}, 0.407 \mathrm{mmol})$. The residue was purified by flash chromatography (gradient elution, 20$30 \%$ AcOEt in hexanes) giving a mixture of the alcohols 5b and $\mathbf{6 b}(3: 1,0.043 \mathrm{~g}, 0.210 \mathrm{mmol}, 49 \%)$ and of the esters 7b and $\mathbf{8 b}(2: 1,0.021 \mathrm{~g}, 0.085 \mathrm{mmol}, 20 \%)$. Mixture of the isomeric alcohols 1-(1,2-dihydro-6methoxynaphthalen-4-yl)ethanol (5b) and (Z)-1ethylidene-1,2,3,4-tetrahydro-7-methoxynaphthalen-2-ol (6b): Pale yellow oil; IR (film) $v_{\max } / \mathrm{cm}^{-1}: 1045,1248$, 1494, 3400; ${ }^{1} \mathrm{H}$ NMR (300 MHz, $\left.\mathrm{CDCl}_{3}\right) \delta$ (5b) 1.45 (d, $J 6.3 \mathrm{~Hz}, 3 \mathrm{H}), 1.8$ (br s, $1 \mathrm{H}), 2.22-2.28(\mathrm{~m}, 2 \mathrm{H}), 2.65$ (t, $J 8.1 \mathrm{~Hz}, 2 \mathrm{H}), 3.79$ (s, 3H), 4.85 (q, J $6.0 \mathrm{~Hz}, 1 \mathrm{H}), 6.19$ $(\mathrm{t}, J 4.8 \mathrm{~Hz}, 1 \mathrm{H}), 6.69(\mathrm{dd}, J 2.7$ and $8.1 \mathrm{~Hz}, 1 \mathrm{H}), 6.98$ (d, J $2.4 \mathrm{~Hz}, 1 \mathrm{H}), 7.07$ (d, J $4.8 \mathrm{~Hz}, 1 \mathrm{H}$ ); (6b) 1.8 (br s, $1 \mathrm{H}), 1.95$ (d, J 7.2 Hz, 3H), 2.55-2.60 (m, 2H), 2.95$3.05(\mathrm{~m}, 2 \mathrm{H}), 3.81$ (s, 3H), 4.99, (t, J $3.3 \mathrm{~Hz}, 1 \mathrm{H}), 6.25$ $(\mathrm{q}, J 7.2 \mathrm{~Hz}, 1 \mathrm{H}), 6.73(\mathrm{dd}, J 2.4$ and $8.1 \mathrm{~Hz}, 1 \mathrm{H}), 7.01-$ $7.10(\mathrm{~m}, 2 \mathrm{H}) ;{ }^{13} \mathrm{C} \mathrm{NMR}\left(75 \mathrm{MHz}, \mathrm{CDCl}_{3}\right) \delta(\mathbf{5 b}+\mathbf{6 b})$ 13.8, 22.6, 23.1, 23.5, 27.3, 30.3, 55.3, 63.8, 67.4, 108.6, $109.7,111.2,113.3,123.2,124.5,128.5,129.0,129.6$, 134.2, 135.2, 140.3, 158.1, 158.2; LRMS (EI) $\mathrm{m} / z$ (rel. int.) $204\left(\mathrm{M}^{+}, 100 \%\right), 144$ (44), 159 (51); HRMS [ESI 
(-)] Calc. for $\left[\mathrm{C}_{13} \mathrm{H}_{16} \mathrm{O}_{2}-\mathrm{H}\right]^{-}$203.1072. Found 203.1077. Mixture of the isomeric esters 1-(1,2-dihydro-6methoxynaphthalen-4-yl)ethyl acetate (7b) and (Z)-1ethylidene-1,2,3,4-tetrahydro-7-methoxynaphthalen-2-yl acetate (8b): Pale yellow oil; IR (film) $v_{\max } / \mathrm{cm}^{-1}: 1042$, 1247, 1730; ${ }^{1} \mathrm{H}$ NMR $\left(300 \mathrm{MHz}, \mathrm{CDCl}_{3}\right) \delta(7 \mathbf{b}) 1.49(\mathrm{~d}$, $J 6.6 \mathrm{~Hz}, 3 \mathrm{H}), 2.08$ (s, 3H), 2.24-2.31 (m, $2 \mathrm{H}), 2.65-$ $2.70(\mathrm{~m}, 2 \mathrm{H}), 3.79(\mathrm{~s}, 3 \mathrm{H}), 5.92(\mathrm{dq}, J 0.6$ and $5.7 \mathrm{~Hz}$, $1 \mathrm{H}), 6.20(\mathrm{t}, J 4.5 \mathrm{~Hz}, 1 \mathrm{H}), 6.71(\mathrm{dd}, J 2.4$ and $8.1 \mathrm{~Hz}$, $1 \mathrm{H}), 6.91(\mathrm{~d}, J 2.4 \mathrm{~Hz}, 1 \mathrm{H}), 7.07(\mathrm{~d}, J 8.4 \mathrm{~Hz}, 1 \mathrm{H}) ;(\mathbf{8 b})$ $1.92(\mathrm{~d}, J 7.2 \mathrm{~Hz}, 3 \mathrm{H}), 2.02$ (s, 3H), 2.16-2.22 (m, 2H), 2.86-2.98 (m, 2H), $3.81(\mathrm{~s}, 3 \mathrm{H}), 6.09(\mathrm{t}, J 3.3 \mathrm{~Hz}, 1 \mathrm{H})$, $6.29(\mathrm{q}, J 6.9 \mathrm{~Hz}, 1 \mathrm{H}), 6.76(\mathrm{dd}, J 2.7$ and $8.7 \mathrm{~Hz}, 1 \mathrm{H})$, 7.02-7.08 (m, 2H); ${ }^{13} \mathrm{C}$ NMR (75 MHz, $\left.\mathrm{CDCl}_{3}\right) \delta(7 \mathbf{b})$ 19.7, 21.4, 23.2, 27.1, 55.3, 69.9, 109.4, 111.6, 126.9, 128.3, 129.5, 133.7, 136.5, 158.2, 170.3; (8b) 13.9, 21.2, 23.9, 28.0, 55.2, 66.8, 108.4, 113.4, 125.1, 128.8, 129.5, 133.1, 134.9, 158.1, 170.6; LRMS (EI) $\mathrm{m} / z$ (rel. int.) $246\left(\mathrm{M}^{+}, 8\right), 186(62), 171(100)$; HRMS [ESI(+)] Calc. for $\left[\mathrm{C}_{15} \mathrm{H}_{18} \mathrm{O}_{3}+\mathrm{H}\right]^{+}$247.1334. Found: 247.1339.

Oxidation of $4 \boldsymbol{c}$ by 1.1 equiv. of TTN. The oxidation was performed following the general procedure, but using $4 c(0.409 \mathrm{~g}, 1.66 \mathrm{mmol}), \mathrm{AcOH} / \mathrm{H}_{2} \mathrm{O}(2: 1,8.3 \mathrm{~mL})$ and TTN. $3 \mathrm{H}_{2} \mathrm{O}(0.812 \mathrm{~g}, 1.83 \mathrm{mmol})$. The residue was purified by flash chromatography (gradient elution, 15$30 \%$ AcOEt in hexanes) giving the alcohol 5c $(0.112 \mathrm{~g}$, $0.547 \mathrm{mmol}, 33 \%$ ), a mixture of the esters $7 \mathbf{c}$ and $\mathbf{8 c}$ $(2: 1,0.076 \mathrm{~g}, 0.309 \mathrm{mmol}, 19 \%)$ and the indan $9 \mathbf{c}(0.094$ g, $0.427 \mathrm{mmol}, 26 \%)$. 1-(1,2-Dihydro-7-methoxynaphthalen-4-yl)ethanol (5c): Colorless oil; IR (film) $v_{\max } / \mathrm{cm}^{-1}: 827,1251,1603,3416 ;{ }^{1} \mathrm{H}$ NMR $(300 \mathrm{MHz}$, $\left.\mathrm{CDCl}_{3}\right) \delta 1.45(\mathrm{~d}, J 6.3 \mathrm{~Hz}, 3 \mathrm{H}), 2.23-2.29(\mathrm{~m}, 2 \mathrm{H}), 2.70$ $(\mathrm{t}, J 8.1 \mathrm{~Hz}, 2 \mathrm{H}), 3.71-3.75(\mathrm{~m}, 1 \mathrm{H}), 3.80(\mathrm{~s}, 3 \mathrm{H}), 4.86$ (q, $J 6.6 \mathrm{~Hz}, 1 \mathrm{H}), 6.04(\mathrm{dt}, J 1.2$ and $4.8 \mathrm{~Hz}, 1 \mathrm{H}), 6.71-$ $6.74(\mathrm{~m}, 2 \mathrm{H}), 7.33(\mathrm{~d}, J 9.3 \mathrm{~Hz}, 1 \mathrm{H}) ;{ }^{13} \mathrm{C} \mathrm{NMR}(75 \mathrm{MHz}$, $\left.\mathrm{CDCl}_{3}\right) \delta 22.7,22.8,28.8,55.3,67.6,111.0,114.1$, 121.4, 124.2, 126.4, 138.9, 140.1, 158.4; LRMS (EI) m/z (rel. int.) 204 ( $\left.\mathrm{M}^{+}, 100 \%\right), 189$ (27), 159 (98); HRMS [ESI(-)] Calc. for $\left[\mathrm{C}_{13} \mathrm{H}_{16} \mathrm{O}_{2}-\mathrm{H}\right]^{-}$203.1072. Found, 203.1077. Mixture of the isomeric esters 1-(1,2-dihydro7-methoxynaphthalen-4-yl)ethyl acetate (7c) and (Z)-1ethylidene-1,2,3,4-tetrahydro-6-methoxynaphthalen-2-yl acetate (8c): pale yellow oil; IR (film) $v_{\max } / \mathrm{cm}^{-1}: 1039$, 1249, 1497, 1732; ${ }^{1} \mathrm{H}$ NMR (300 MHz, $\left.\mathrm{CDCl}_{3}\right) \delta(\mathbf{7 c})$ 1.47 (d, J $6.6 \mathrm{~Hz}, 3 \mathrm{H}), 2.07$ (s, 3H), 2.22-2.31 (m, 2H), 2.67-2.75 (m, 2H), $3.80(\mathrm{~s}, 3 \mathrm{H}), 5.89(\mathrm{q}, J 6.3 \mathrm{~Hz}, 1 \mathrm{H})$, $6.04(\mathrm{t}, J 3.3 \mathrm{~Hz}, 1 \mathrm{H}), 6.70-6.76(\mathrm{~m}, 2 \mathrm{H}), 7.23$ (d, J 9.3 $\mathrm{Hz}, 1 \mathrm{H}) ;(8 \mathrm{c}) 1.88(\mathrm{~d}, J 7.2 \mathrm{~Hz}, 3 \mathrm{H}), 2.62-2.66(\mathrm{~m}, 2 \mathrm{H})$, 2.93-3.04 (m, 2H), 3.80 (s, 3H), 6.08 (t, J $3.0 \mathrm{~Hz}, 1 \mathrm{H})$, $6.17(\mathrm{q}, J 7.2 \mathrm{~Hz}, 1 \mathrm{H}), 6.64(\mathrm{~d}, J 2.4 \mathrm{~Hz}, 1 \mathrm{H}), 6.71-6.76$ $(\mathrm{m}, 1 \mathrm{H}), 7.48(\mathrm{~d}, J 9.0 \mathrm{~Hz}, 1 \mathrm{H}) ;{ }^{13} \mathrm{C}$ NMR $(75 \mathrm{MHz}$, $\left.\mathrm{CDCl}_{3}\right) \delta(7 \mathrm{c}) 19.9,21.5,22.9,28.7,55.3,70.2,111.1$, $114.2,123.8,124.2,126.0,136.4,138.7,158.6,170.6$. (8c) 13.9, 21.4, 25.3, 27.9, 55.3, 67.2, 113.0, 113.1, 122.8, 125.3, 127.1, 132.7, 137.2, 158.7, 170.6; LRMS (EI) $\mathrm{m} / \mathrm{z}$ (rel. int.): $246\left(\mathrm{M}^{+}, 21 \%\right), 186$ (100), 171 (11); HRMS [ESI(+)] Calc. for $\left[\mathrm{C}_{15} \mathrm{H}_{18} \mathrm{O}_{3}+\mathrm{H}\right]^{+} 247.1334$. Found: 247.1332. 1-(2,3-Dihydro-5-methoxy-1H-inden1-yl)-2-hydroxypropan-1-one (9c): pale yellow oil; IR (film) $v_{\max } / \mathrm{cm}^{-1}: 1152,1255,1706,3510 ;{ }^{1} \mathrm{H}$ NMR (300 $\left.\mathrm{MHz}, \mathrm{CDCl}_{3}\right) \delta 1.58(\mathrm{~d}, J 7.2 \mathrm{~Hz}, 3 \mathrm{H}), 1.65-1.77(\mathrm{~m}$, $1 \mathrm{H}), 2.34-2.50(\mathrm{~m}, 1 \mathrm{H}), 2.93-2.98(\mathrm{~m}, 2 \mathrm{H}), 3.50(\mathrm{br} \mathrm{s}$, 1H), 3.81 (s, 3H), 3.87 (q, J $7.8 \mathrm{~Hz}, 1 \mathrm{H}), 4.29$ (dt, J 3.0 and $10.2 \mathrm{~Hz}, 1 \mathrm{H}), 6.72-6.81(\mathrm{~m}, 2 \mathrm{H}), 7.13(\mathrm{~d}, J 9.3 \mathrm{~Hz}$, $1 \mathrm{H}) ;{ }^{13} \mathrm{C} \mathrm{NMR}\left(75 \mathrm{MHz}, \mathrm{CDCl}_{3}\right) \delta 15.3,29.6,34.2,48.8$, 55.4, 74.2, 111.8, 116.0, 128.3, 129.1, 141.0, 159.1, 212.6; LRMS (EI) $\mathrm{m} / z$ (rel. int.) $220\left(\mathbf{M}^{+}, 11 \%\right), 175$ (100), 147 (72); HRMS [ESI(-)] Calc. for $\left[\mathrm{C}_{13} \mathrm{H}_{16} \mathrm{O}_{3}-\right.$ H] 219.1021. Found: 219.1023.

Oxidation of $4 c$ by 1.7 equiv. TTN. The oxidation was performed following the general procedure, but using $\mathbf{4 c}$ $(0.130 \mathrm{~g}, 0.530 \mathrm{mmol})$ in $\mathrm{AcOH} / \mathrm{H}_{2} \mathrm{O}(2: 1,3.0 \mathrm{~mL})$ and TTN. $3 \mathrm{H}_{2} \mathrm{O}(0.397 \mathrm{~g}, 0.893 \mathrm{mmol})$. The residue was purified by flash chromatography (gradient elution, 20$40 \%$ AcOEt in hexanes) giving a mixture of the esters $7 \mathbf{c}$ and $8 \mathbf{c}(2: 1,0.014 \mathrm{~g}, 0.055 \mathrm{mmol}, 10 \%)$ and the indan $9 \mathbf{c}$ (0.049 g, $0.224 \mathrm{mmol}, 42 \%)$.

1-(2,3-Dihydro-1H-inden-3-yl)-3-hydroxy-3-methylbutan-1-one $(\mathbf{1 7 d})$. The oxidation was performed following the general procedure, using $\mathbf{4 d}(0.108 \mathrm{~g}, 0.532$ $\mathrm{mmol}), \mathrm{AcOH} / \mathrm{H}_{2} \mathrm{O}(2: 1,2.4 \mathrm{~mL})$ and TTN. $3 \mathrm{H}_{2} \mathrm{O}(0.355$ $\mathrm{g}, 0.798 \mathrm{mmol}$ ). The residue was purified by flash chromatography (gradient elution, 20-30\% AcOEt in hexanes) giving the indan $\mathbf{1 7 d}(0.073 \mathrm{~g}, 0.335 \mathrm{mmol}$, 63\%). Colorless oil; IR (film) $v_{\max } / \mathrm{cm}^{-1}: 758,1154,1698$, 3437; ${ }^{1} \mathrm{H}$ NMR (300 MHz, $\mathrm{CDCl}_{3}$ ) $\delta 1.21$ (s, 3H), 1.22 (s, 3H), 2.06 (s, 1H), 2.28-2.36 (m, 2H), 2.64 (d, J 17.4 $\mathrm{Hz}, 1 \mathrm{H}), 2.73(\mathrm{~d}, J 17.4 \mathrm{~Hz}, 1 \mathrm{H}), 2.97$ (td, $J 7.2$ and $15.6 \mathrm{~Hz}, 1 \mathrm{H}), 3.06(\mathrm{dt}, J 8.4$ and $17.4 \mathrm{~Hz}, 1 \mathrm{H}), 4.08(\mathrm{dd}$, $J 6.6$ and $7.8 \mathrm{~Hz}, 1 \mathrm{H}), 7.16-7.29(\mathrm{~m}, 4 \mathrm{H}) ;{ }^{13} \mathrm{C}$ NMR $(75$ $\left.\mathrm{MHz}, \mathrm{CDCl}_{3}\right) \delta 28.4,29.1,29.2,31.8,50.6,59.2,69.8$, 124.7, 125.0, 126.5, 127.7, 140.1, 144.5, 213.1; LRMS (EI) $\mathrm{m} / \mathrm{z}$ (rel. int.) $218\left(\mathrm{M}^{+}, 1 \%\right), 144$ (11), 117 (100); HRMS [ESI(+)] Calc. for $\left[\mathrm{C}_{14} \mathrm{H}_{18} \mathrm{O}_{2}+\mathrm{Na}\right]^{+} 241.1205$. Found 241.1221.

1-(2,3-Dihydro-6-methoxy-1H-inden-3-yl)-3-hydroxy-3methylbutan-1-one (17e). The oxidation was performed following the general procedure, using $\mathbf{4 e}(0.091 \mathrm{~g}, 0.390$ 
$\mathrm{mmol}), \mathrm{AcOH} / \mathrm{H}_{2} \mathrm{O}(2: 1,2.1 \mathrm{~mL})$ and TTN.3 $\mathrm{H}_{2} \mathrm{O}(0.260$ $\mathrm{g}, 0.590 \mathrm{mmol})$. The residue was purified by flash chromatography (gradient elution, 25-30\% AcOEt in hexanes) giving the indan $17 \mathrm{e}(0.057 \mathrm{~g}, 0.231 \mathrm{mmol}, 59 \%)$ and the starting material $4 \mathrm{e}(0.004 \mathrm{~g}, 0.017 \mathrm{mmol}, 4 \%)$. Colorless oil; IR (film) $v_{\max } / \mathrm{cm}^{-1}: 1033,1462,1698,3477$; ${ }^{1} \mathrm{H}$ NMR (300 MHz, $\left.\mathrm{CDCl}_{3}\right) \delta 1.21(\mathrm{~s}, 6 \mathrm{H}), 2.03(\mathrm{~s}, 1 \mathrm{H})$, 2.24-2.37 (m, 2H), 2.62 (d, J $17.4 \mathrm{~Hz}, 1 \mathrm{H}), 2.70$ (d, J $17.4 \mathrm{~Hz}, 1 \mathrm{H}), 2.88(\mathrm{td}, J 7.5$ and $15.3 \mathrm{~Hz}, 1 \mathrm{H}), 2.97(\mathrm{td}, J$ 7.5 and $15.3 \mathrm{~Hz}, 1 \mathrm{H}), 3.77(\mathrm{~s}, 3 \mathrm{H}), 4.03$ (dd, $J 8.1$ and $14.4 \mathrm{~Hz}, 1 \mathrm{H}), 6.74-6.79$ (m. 2H), 7.15 (d, J 7.5 Hz, 1H); ${ }^{13} \mathrm{C} \mathrm{NMR}\left(75 \mathrm{MHz}, \mathrm{CDCl}_{3}\right) \delta 28.8,29.1,29.2,30.8,50.4$, 55.3, 59.3, 69.6, 110.1, 113.6, 125.3, 136.3, 141.4, 158.6, 213.0; LRMS (EI) $\mathrm{m} / z$ (rel. int.) $248\left(\mathrm{M}^{+}, 6 \%\right), 174$ (10), 147 (100); HRMS [ESI(+)] Calc. for $\left[\mathrm{C}_{15} \mathrm{H}_{20} \mathrm{O}_{3}+\mathrm{Na}\right]^{+}$ 271.1310. Found: 271.1304.

1-(2,3-Dihydro-5-methoxy-1H-inden-1-yl)-3-hydroxy-3methylbutan-1-one (17f). The oxidation was performed following the general procedure, using $4 \mathbf{f}(0.075,0.325$ $\mathrm{mmol}), \mathrm{AcOH} / \mathrm{H}_{2} \mathrm{O}(2: 1,1.5 \mathrm{~mL})$ and TTN.3 $\mathrm{H}_{2} \mathrm{O}(0.216$ $\mathrm{g}, 0.487 \mathrm{mmol})$. The residue was purified by flash chromatography (gradient elution, 20-30\% AcOEt in hexanes) giving the indan $\mathbf{1 7 f}(0.050 \mathrm{~g}, 0.202 \mathrm{mmol}, 62 \%)$. Colorless oil; IR (film) $v_{\max } / \mathrm{cm}^{-1}: 811,1260,1699,3490$; ${ }^{1} \mathrm{H}$ NMR $\left(300 \mathrm{MHz}, \mathrm{CDCl}_{3}\right) \delta 1.20(\mathrm{~s}, 3 \mathrm{H}), 1.21(\mathrm{~s}, 3 \mathrm{H})$, 2.82-2.47 (m, 2H), 2.62 (d, J $17.4 \mathrm{~Hz}, 1 \mathrm{H}), 2.71$ (d, $J$ $17.4 \mathrm{~Hz}, 1 \mathrm{H}), 2.94(\mathrm{td}, J 8.4$ and $15.9 \mathrm{~Hz}, 1 \mathrm{H}), 3.04$ (dt, $J$ 7.8 and $15.9 \mathrm{~Hz}, 1 \mathrm{H}), 3.79$ (s, 3H), 4.00 (dd, $J 6.0$ and 7.8 $\mathrm{Hz}), 6.72-6.76$ (m, 1H), 6.84 (d, J 2.1 Hz, 1H), 7.13 (d, J $8.4 \mathrm{~Hz}, 1 \mathrm{H}) ;{ }^{13} \mathrm{C} \mathrm{NMR}\left(75 \mathrm{MHz}, \mathrm{CDCl}_{3}\right) \delta 29.0,29.5$, 29.6, 32.3, 50.6, 55.6, 58.7, 69.9, 110.5, 112.9, 125.6, 132.4, 146.5, 160.0, 213.8; LRMS (EI) $\mathrm{m} / \mathrm{z}$ (rel. int.) 248 $\left(\mathrm{M}^{+}, 4 \%\right), 174$ (100), 91 (11); Anal. calc. for $\mathrm{C}_{15} \mathrm{H}_{20} \mathrm{O}_{3}: \mathrm{C}$, 72.55; H, 8.12. Found: C, 72.53; H, 7.92.

trans-1-(2,3-Dihydro-1-methyl-1H-inden-3-yl)-3-hydroxy3-methylbutan-1-one $(\mathbf{1 7 g})$. The oxidation was performed following the general procedure, using $\mathbf{4 g}(0.104 \mathrm{~g}, 0.481$ $\mathrm{mmol}), \mathrm{AcOH} / \mathrm{H}_{2} \mathrm{O}(2: 1,2.4 \mathrm{~mL})$ and TTN.3 $\mathrm{H}_{2} \mathrm{O}(0.321$ $\mathrm{g}, 0.722 \mathrm{mmol})$. The residue was purified by flash chromatography (gradient elution, 25-30\% AcOEt in hexanes) giving the indan $\mathbf{1 7 g}(0.072 \mathrm{~g}, 0.312 \mathrm{mmol}, 65 \%)$ and the starting material $\mathbf{4 g}(0.004 \mathrm{~g}, 0.019 \mathrm{mmol}, 4 \%)$. Pale yellow oil; IR (film) $v_{\max } / \mathrm{cm}^{-1}: 758,1376,1765,3437$; ${ }^{1} \mathrm{H} \mathrm{NMR}\left(300 \mathrm{MHz}, \mathrm{CDCl}_{3}\right) \delta 1.18$ (s, 3H), 1.20 (s, 3H), $1.29(\mathrm{~d}, J 7.5 \mathrm{~Hz}, 3 \mathrm{H}), 1.87$ (dt, $J 12.9$ and $8.1 \mathrm{~Hz}), 2.52-$ $2.59(\mathrm{~m}, 1 \mathrm{H}), 2.63(\mathrm{~d}, J 16.8 \mathrm{~Hz}), 2.73(\mathrm{~d}, J 16.8 \mathrm{~Hz})$, 3.39 (sx, J 7.2 Hz, 1H), 4.07 (dd, J 3.0 and $8.7 \mathrm{~Hz}, 1 \mathrm{H})$, 7.17-7.31 (m, 4H); $\left.{ }^{13} \mathrm{C} \mathrm{NMR} \mathrm{(75} \mathrm{MHz}, \mathrm{CDCl}_{3}\right) \delta 20.2$, 29.2, 29.3, 37.5, 38.4, 50.6, 58.1, 70.0, 124.0, 124.8, 126.7,
128.0, 139.7, 149.2, 213.0; LRMS (EI) $m / z$ (rel. int.) 214 (4\%), 131 (100), 115 (29); HRMS [ESI(+)] Calc. for $\left[\mathrm{C}_{15} \mathrm{H}_{20} \mathrm{O}_{2}+\mathrm{Na}\right]^{+}$255.1361. Found: 255.1350.

\section{Acknowledgments}

The authors wish to thank FAPESP, CNPq and CAPES for their continuous financial support. M. V. Craveiro is acknowledged for performing some initial experiments.

\section{References}

1. For some reviews concerning thallium(III) chemistry, see: McKillop, A.; Taylor, E. C. In Comprehensive Organometallic Chemistry; Wilkinson, G., ed.; Pergamon Press: New York, 1982, vol. 7, p. 465; Ferraz, H. M. C.; Silva Jr., L. F.; Vieira, T. de O.; Synthesis 1999, 2001.

2. Byrd, J. E.; Halpern, J.; J. Am. Chem. Soc. 1973, 95, 2586.

3. Ferraz, H. M. C.; Brocksom, T. J.; Pinto, A. C.; Abla, M. A.; Zocher, D. T. H.; Tetrahedron Lett. 1986, $27,811$.

4. Ferraz, H. M.; Ribeiro, C. M. R.; Grazini, M. V. A.; Brocksom, T. J.; Brocksom, U.; Tetrahedron Lett. 1994, 35, 1497.

5. Ferraz, H. M. C.; Grazini, M. V. A.; Ribeiro, C. M. R.; Brocksom, U.; Brocksom, T. J.; J. Org. Chem. 2000, 65, 2606.

6. Ferraz, H. M. C.; Longo Jr., L. S.; Zukerman-Schpector, J.; J. Org. Chem. 2002, 67, 3518.

7. Silva Jr., L. F.; Craveiro, M. V.; Molecules 2005, 10, 1419.

8. Ferraz, H. M. C.; Santos, A. P.; Silva Jr., L. F.; Vieira, T. de O.; Synth. Commun. 2000, 30, 751.

9. Ferraz, H. M. C.; Silva Jr., L. F.; Tetrahedron 2001, 57, 9939.

10. Kocovský, P.; Pour, M.; J. Org. Chem. 1990, 55, 5580.

11. Kocovský, P.; Langer, V.; Gogoll, A.; J. Chem. Soc., Chem. Commun. 1990, 1026.

12. Kocovský, P.; Baines, R. S.; Tetrahedron Lett. 1993, 34, 6139.

13. Kocovský, P.; Baines, R. S.; J. Org. Chem. 1994, 59, 5439.

14. Corey, E. J.; Snider, B. B.; J. Am. Chem. Soc. 1972, 94, 2549.

15. Although 6 equivalents of methyllithium were used in these reactions, the corresponding methyl ketones were always obtained as a byproduct.

16. Ferraz, H. M. C.; Grazini, M. V. A.; Silva Jr., L. F.; Longo Jr., L. S.; Synth. Commun. 1999, 29, 1953.

17. Ferraz, H. M. C.; Aguilar, A. M.; Silva Jr., L. F.; Synthesis 2003, 1031.

18. Silva Jr., L. F.; Sousa, R. M. F.; Ferraz, H. M. C.; Aguilar, A. M.; J. Braz. Chem. Soc. 2005, 16, 1160.

19. Preite, M. D.; Cuellar, M. A.; Chem. Commun. 2004, 1970.

Received: May 3, 2006

Published on the web: July 13, 2006

FAPESP helped in meeting the publication costs of this article. 